\title{
Paper
}

\section{Stochastic resonance in a pulse-type cell body model}

\author{
Diana Elizabeth Jimenez Bejarano ${ }^{1}$ and Katsutoshi Saeki ${ }^{1 a)}$ \\ ${ }^{1}$ College of Science and Technology, Nihon University \\ 24-1, Narashinodai 7-chome, Funabashi-shi, Chiba 274-8501, Japan \\ a) saeki.katsutoshi@nihon-u.ac.jp
}

Received January 24, 2017; Revised May 29, 2017; Published October 1, 2017

\begin{abstract}
In this work, we study the presence of stochastic resonance (SR) in a hardware pulsetype cell body model. SR is a phenomenon that uses noise to boost weak periodic signals. For this purpose, we design a random noise source and include it in the model because it has been demonstrated that adding random noise to a pulse-type cell body model significantly improves its output signal-to-noise ratio (SNR). The noise also reduces the voltage needed for circuit oscillation. Finally, a decrement in the delay time is observed in the presence of noise.
\end{abstract}

Key Words: stochastic resonance (SR), noise, pulse-type cell body model, analog circuit, negative resistance, signal-to-noise ratio (SNR)

\section{Introduction}

Stochastic resonance (SR) is a phenomenon that can be observed when noise assists the improvement of a nonlinear system's output signal [1]. Several biological studies have established the presence of SR. Most of them have demonstrated the benefits of noise in nonlinear systems, creating opportunities for possible uses in medicine, as summarized by Hanggi in his survey [2]. Other studies on the human brain, such as the one by Ward et al., suggest that noise influences neural synchrony, which contributes to the communication of information between neurons [3]. Similarly, the presence of SR has been observed in the visual cortex [4]. These studies use a signal-to-noise ratio (SNR) graph to measure the effect of noise in a system and to characterize SR [5,6]. Electronic studies show that noise can be used for improving analog circuit response [7-9]. Based on the above, adding noise to the system to investigate the existence of SR becomes significant because it could render the proposed circuit more exact to its biological counterpart. Studies have demonstrated that in artificial systems like cochlear implants, the addition of an appropriate amount noise improves device functioning [10]. Some studies aim to analyze the presence of SR in CMOS neuron circuits [11], focusing on the methodology by which the presence of noise causes neuron spikes and discussing the parameters that influence the SR characteristic. However, the proposed cell body model [12] operates differently from the former neuron models because it uses a negative resistance circuit to generate neuron-like pulses. Due to the configuration of the circuit, the output oscillation is not related to the period of the input signal. The present study verifies the presence of SR and confirms that noise enables neuron spikes. Thereafter, it explores the application of noise for improving signal transmission speed and for providing useful employments for inherent and external noises. 
To perform this study, a random noise source is designed. This source is connected to a sine wave source for signal modulation, and the random and sine signals are added to the input voltage. Finally, we analyze the output oscillation and amplitude. The results of "when noise is applied" and "when noise is absent" are compared for analyzing the benefits of noise.

\section{Basic operation of a pulse-type cell body model}

The SR phenomenon is analyzed in a pulse-type cell body model [13] that model uses a $\Lambda$-shaped negative resistance configuration to generate circuit oscillation.

\subsection{Negative resistance circuit}

The $\Lambda$-shaped negative resistance configuration is shown in Fig. 1, where M1 and M2 are the N-type and P-type enhancement MOSFETs, respectively. When a voltage, $\mathrm{Vcm}$, is applied, the voltage difference between $\mathrm{Vcm}$ and Va decreases. However, the voltage applied to the gate of M1 continues to increase, enabling more current to flow through M1, until the transistors enter the linear region and the current decreases. The negative resistance characteristic is depicted in Fig. 2, where the horizontal and vertical axes represent the input voltage, Va and the current flowing through M1, respectively. The region where $\operatorname{Im} 1$ increases is the active region of the negative resistance.

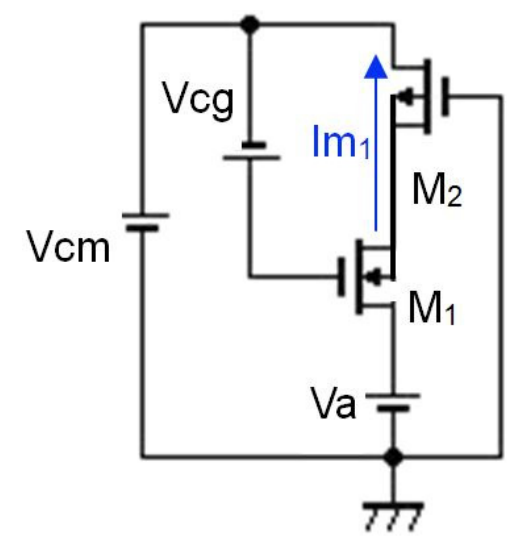

Fig. 1. $\Lambda$-shaped negative resistance circuit.

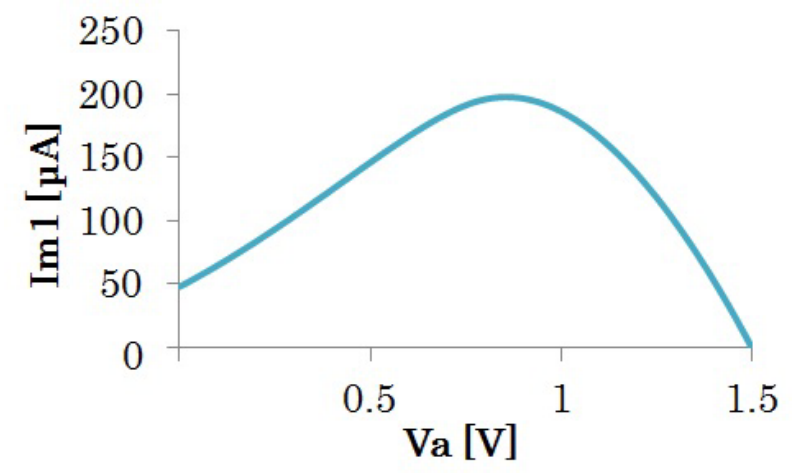

Fig. 2. $\Lambda$-shaped negative resistance characteristic.

\subsection{Pulse-type cell body model}

The purpose of this model is to simulate the behavior of a biological neuron; hence, it is simulated as a pacemaker neuron, i.e., the circuit will generate an output by itself. A specific condition for this design is that the voltage, Va, must be sufficiently high to cause circuit oscillation; i.e., the negative resistance circuit needs a certain voltage (Va) such that current flows through it, to be activated. This current can be seen in Fig. 2. In order to implement this model in VLSI with a minimum area, the values of the capacitors are in the order of fF. The model is displayed in Fig. 3(a), where M1 and M2 form the $\Lambda$-shaped negative resistance. M3, M4, and M5 are the N-type enhancement MOSFETs. M3 provides a steady voltage to the negative resistance, whereas M4 represents the leakage resistance that drives the charge from $\mathrm{Cg}$. $\mathrm{Cg}$ and M5 change the voltage applied to the $\Lambda$-shaped negative resistance. $\mathrm{Cm}$ represents the membrane capacitance of a biological cell and is charged by the current from $\mathrm{Cg}$; the variation in the value of $\mathrm{Cm}$ modifies the output voltage (Vout). This modification causes an increase and decrease in Vout. Hence, the voltage across the $\Lambda$-shaped negative resistance also increases or decreases. By repeating this operation, the cell body model oscillates. The typical oscillation of the circuit is shown in Fig. 3(b).

This circuit was designed to generate oscillations at low capacitance values and this characteristic can be observed in Fig. 4, where the horizontal axis indicates the capacitance and the vertical axis, the frequency. This graph indicates that for low values of $\mathrm{Cm}$ and $\mathrm{Cg}$, the output oscillation is high; the frequency reduces as the capacitance value increases. In Fig. 5, the horizontal axis represents the 


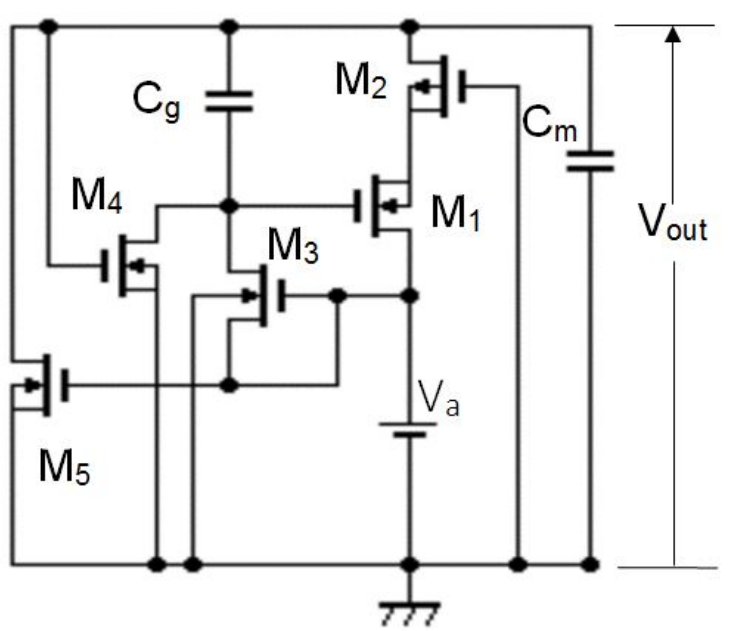

(a) Pulse-type cell body model

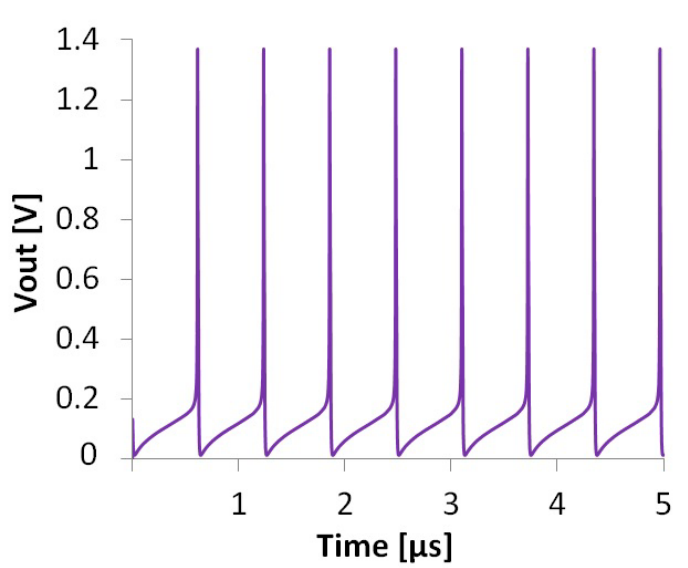

(b) Output waveform

Fig. 3. Pulse-type cell body model circuit and output.

aspect ratio of $\mathrm{M} 1$ and $\mathrm{M} 2$, the transistors that conform to the negative resistance and have the same value. The vertical axis represents the frequency of the pulse-type cell body model. This figure shows that the frequency increases with the increase in the aspect ratio $(\mathrm{W} / \mathrm{L})$. This is due to the amount of current that flows through the negative resistance. As the current flow increases, the negative resistance turns 'ON' faster. Therefore, the switching time for pulses generation also increases and the frequency rises.

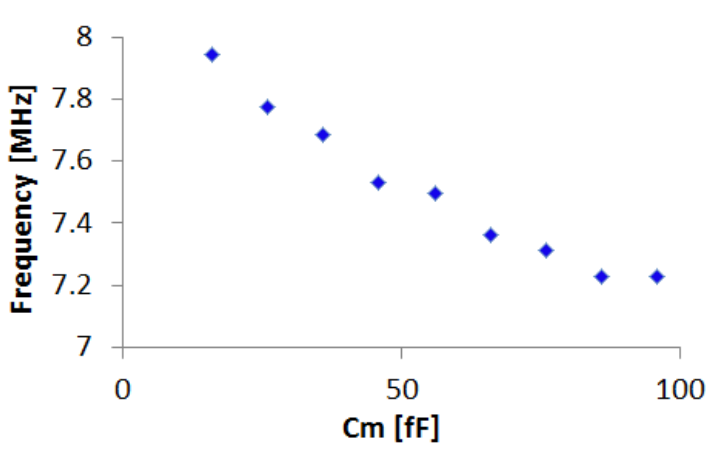

(a) Frequency variation due to $\mathrm{Cm}$

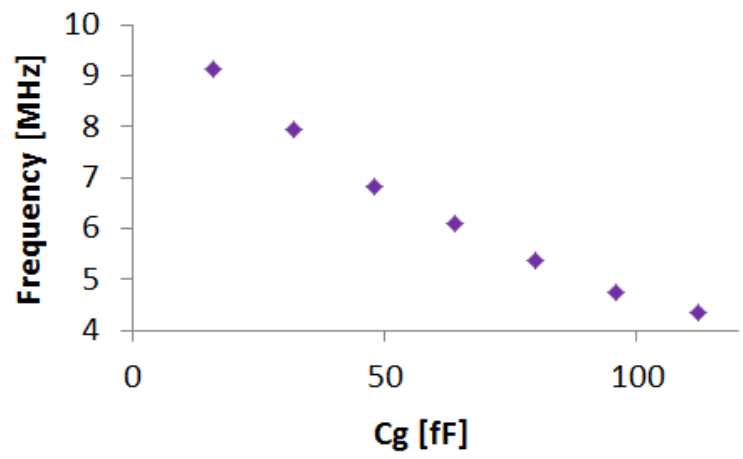

(b) Frequency variation due to $\mathrm{Cg}$

Fig. 4. Capacitance vs. frequency characteristic.

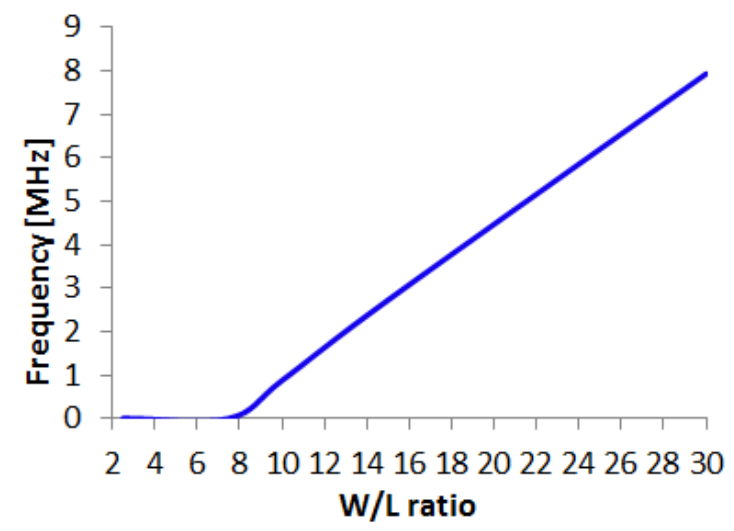

Fig. 5. Capacitance vs. aspect ratio characteristic. 


\section{Presence of stochastic resonance in the pulse-type cell body model}

\subsection{Noise source}

To study the presence of SR in the pulse-type cell body model, a noise source was designed. The noise signal is a random sequence simulated in HSPICE. In order to modulate the noise and create a more variable source, a sine wave source was also added. The sinusoidal source was simulated in the circuit simulator, HSPICE, using the command: $\sin (00.2$ 20meg). Random sequences are composed by evenly distributed random real numbers generated externally using a computer application. The random sequences were added as a piecewise linear (PWL) function. The PWL source was called Vn. The noise source circuit and its output are shown in Fig. 6, where Vs $=0.2 \sin (2 \pi f)$, with a maximum voltage of $0.2[\mathrm{~V}]$ and frequency of $20[\mathrm{MHz}]$. In this figure, the Vn values are random numbers, greater than or equal to zero but less than 0.5.; i.e., the maximum amount of noise $(\mathrm{Vn})$ is $0.5[\mathrm{~V}]$. In the following results, Vn indicates the maximum value of any PWL function used in this study and "Noise source" indicates Vs + Vn.

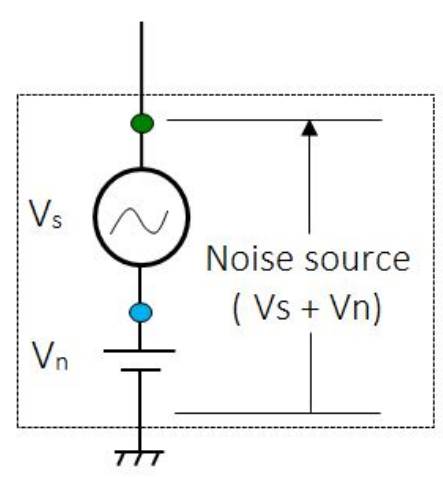

(a) Noise source circuit and connection

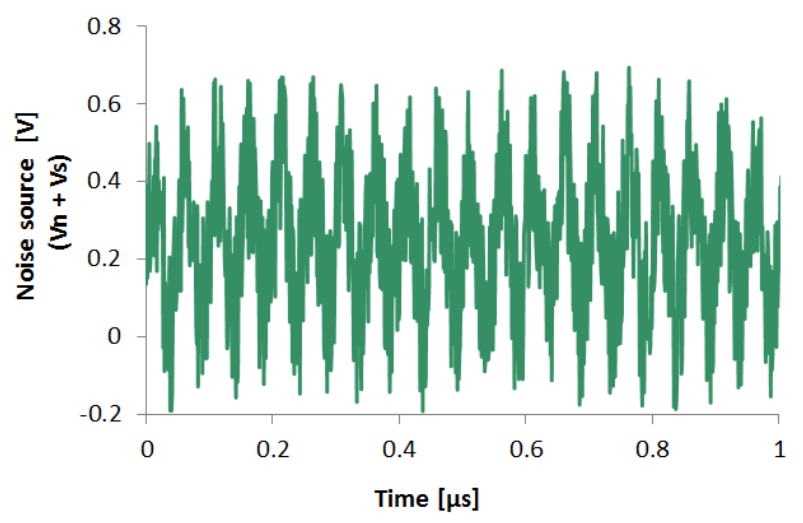

(b) Noise source output

Fig. 6. Noise source circuit and output.

\subsection{Noise addition to the pulse-type cell body model}

In this study, the cell body uses the voltage, Va, depicted in Fig. 3, to power the entire circuit and generate the characteristic output of a neuron [13]. Owing to the occurrence of the SR phenomenon, adding noise to a non-linear system improves the circuit output. As the pulse type cell body model used in this study is a pacemaker neuron, it cannot generate oscillations at a low input voltage (Va). Hence, noise is added to the cell body model's positive bias voltage, Va, as shown in Fig. 7. In this figure, when noise is added, the total input voltage, Vin, will be the sum of Vn, Vs, and Va. When noise is not added to the model, the total input (Vin) remains as Va only. Therefore, the average of the total input voltage (Vin) with noise, differs from that without noise.
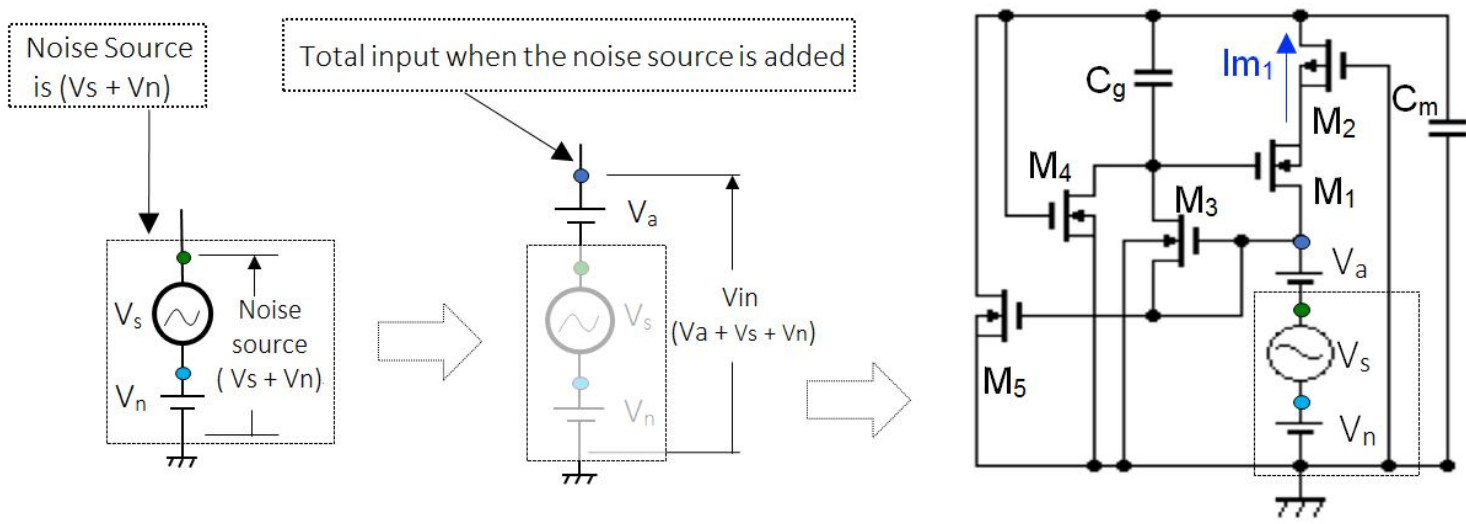

Fig. 7. Low capacitance pulse-type cell body model with noise source. 
The current in the negative resistance, Im1, due to the added noise value, is represented in Fig. 8. This figure demonstrates the effects of the changes in the noise voltage on the current and consequently on the region, where the signal is generated. Simultaneously, the behavior of $\operatorname{Im} 1$ is compared for different values of $\mathrm{W} / \mathrm{L}$ of the negative resistance. It can be seen that although the current peak increases for large $\mathrm{W} / \mathrm{L}$ values, it decreases again after a certain $\mathrm{W} / \mathrm{L}$ value. In Fig. 8, the noise value that marks the highest current peak is $\mathrm{Vn}=1.5[\mathrm{~V}]$.

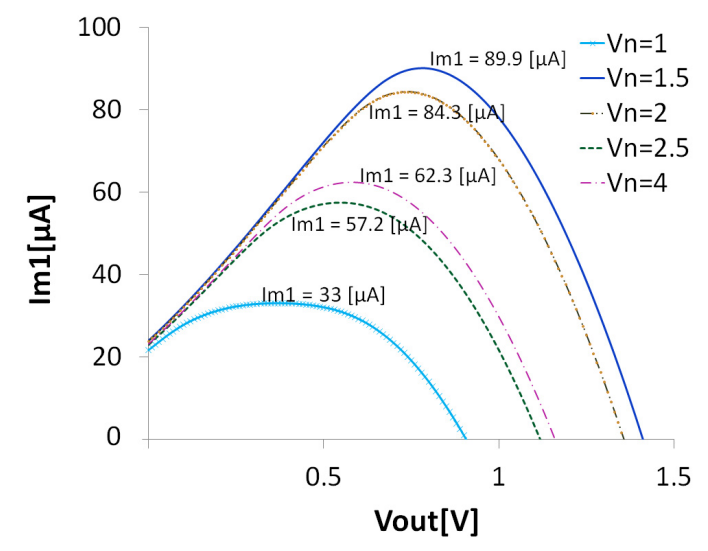

(a) Im1, when the aspect ratio $=7.5$

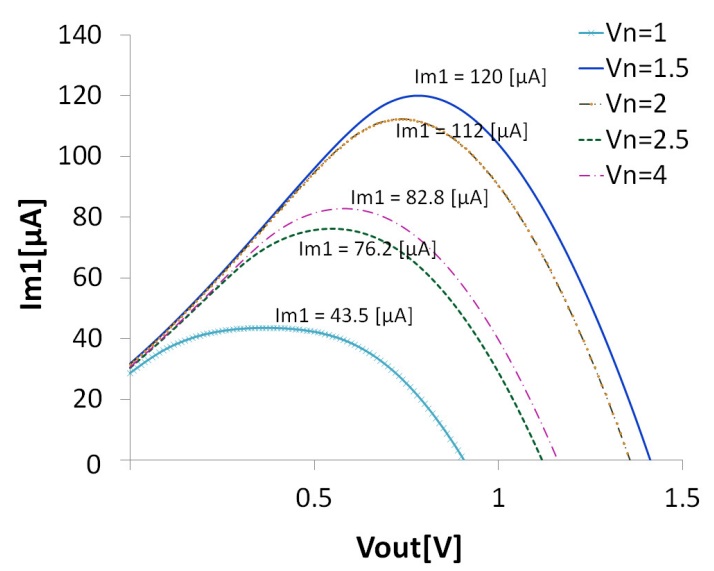

(b) $\operatorname{Im} 1$, when the aspect ratio $=10$

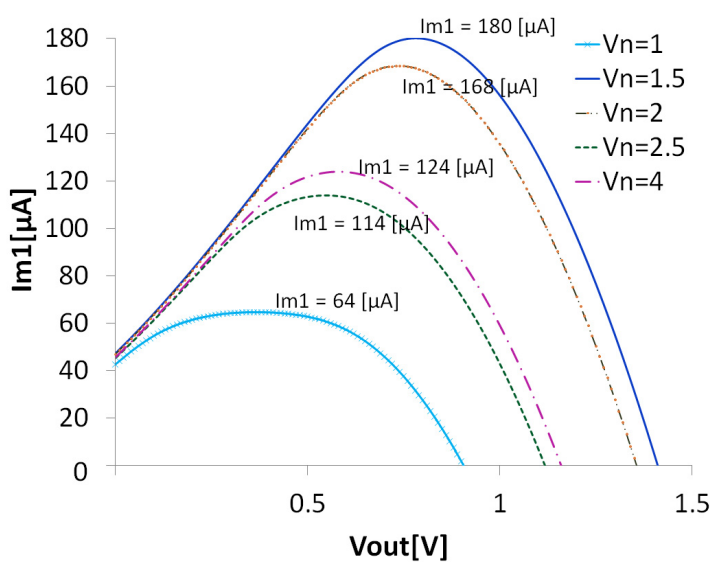

(c) $\operatorname{Im} 1$, when the aspect ratio $=15$

Fig. 8. Current flowing through the negative resistance.

\subsection{Stochastic resonance (SR) characteristic}

A common method of measuring the SR is to calculate the SNR. The result presented in Fig. 9 was calculated with the values obtained from the simulation of the cell body. The standard derivation (represented as $\delta$ ) on the horizontal axis was calculated from each random sequence (Vn). The mean value (represented as $\mu$ ) was calculated from the output peak-to-peak voltage. The vertical axis represents the SNR, determined to be: $\mu / \delta$. The SNR peak shows the appropriate level of noise that should be applied for improving the circuit response [1].

When the width (W) values of M1 and M2 are increased simultaneously, the aspect ratio increases. This causes an increment in the current flow to Cm, amplifying the output value. As the SNR is linearly dependent on the output, the total SNR value increases; i.e., when the output is high the SNR is also high. On the other hand, the SNR characteristics for various values of the frequency and amplitude do not affect the SR characteristics, in general. As observed in Fig. 10 and Fig. 11, the SNR still shows a peak for a nonzero noise value. However, it is important to note that the circuit has a limit for the frequency and amplitude at which it can work. In Fig. 10 and Fig. 11, the SNR variation with the amplitude and frequency of the sine wave source is depicted. It can be observed 


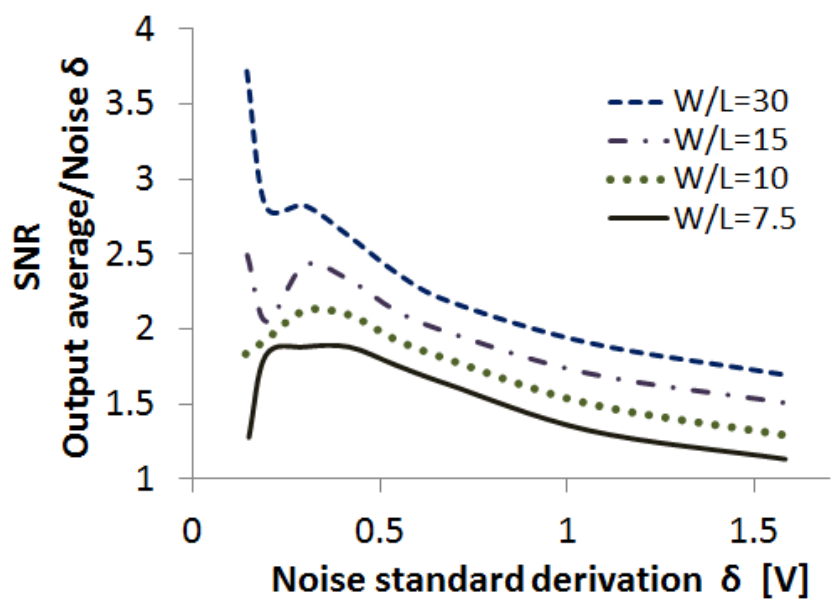

Fig. 9. SNR characteristic.

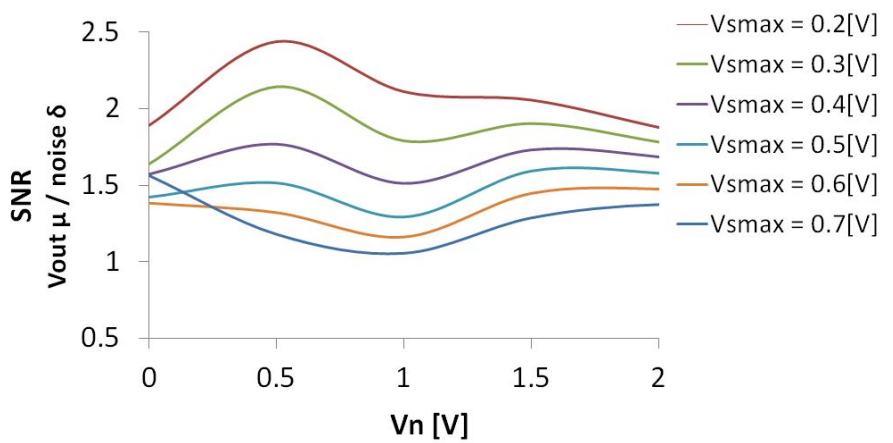

Fig. 10. SNR characteristic for different amplitude values of Vs.

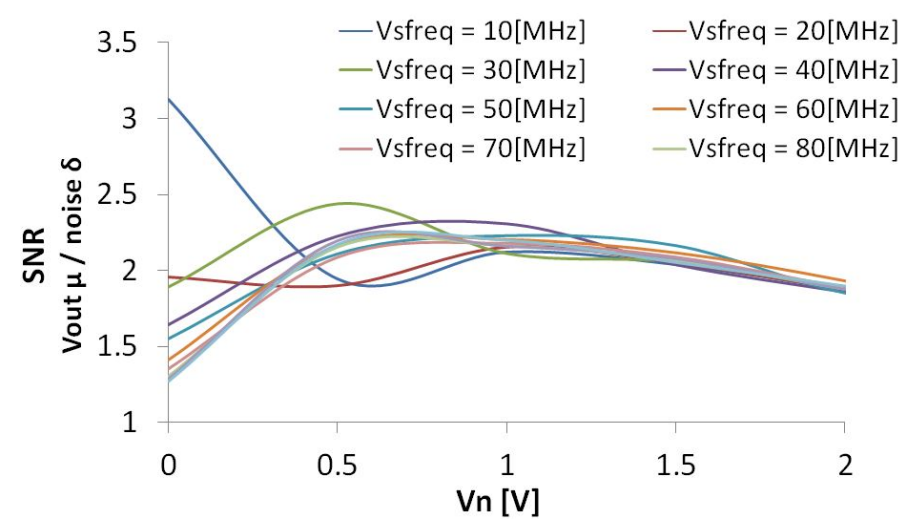

Fig. 11. SNR characteristic for different frequency values of Vs.

that a typical SR bell shape is present, even when the parameters (Vsmax and Vsfreq) are different. Nevertheless, this characteristic is lost when the frequency of the Vs source is high, as shown in Fig. 11. This is because the signal loses its typical random sequence, when the frequency is high. A similar effect is observed for high values of Va, as in Fig. 12. This occurs because the oscillation of the circuit changes in accordance with the negative resistance input voltage, Va.

It was observed that adding only a sinusoidal source is insufficient. The addition of a random noise source, Vn, is essential for the model to oscillate, as observed in Fig. 13 and Fig. 14.

To compare the effects of the noise in the circuit, the aspect ratio values for the negative resistance were fixed at $\mathrm{W} / \mathrm{L}=7.5$. The results are displayed in Fig. 15. Here, Vin is Va + Vs + Vn. Adding noise to the circuit reduces the output rise time in the pulse-type cell body model; this is valid for any noise value. Thus, the reduction in the rise time is directly proportional to the increment in the noise level. However, it can be observed from the output signal that at high noise levels, the signal loses 


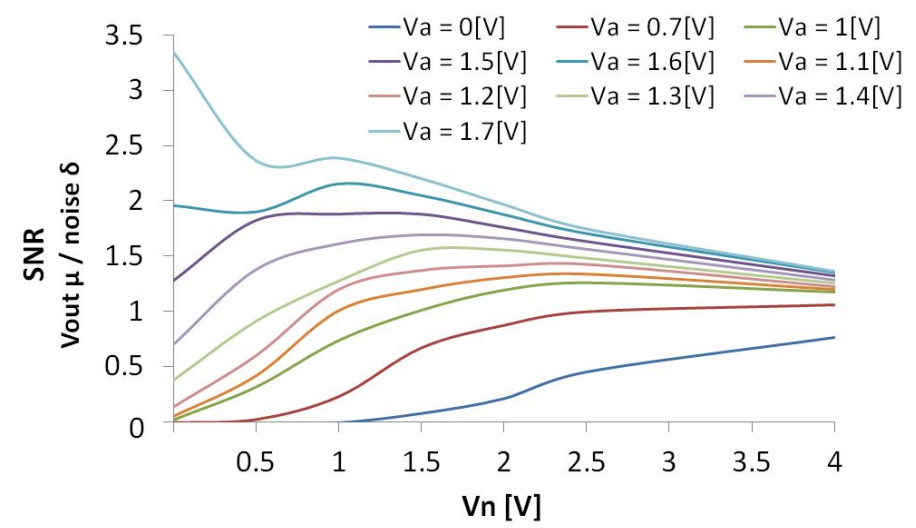

Fig. 12. SNR characteristic for different input voltage Va values.
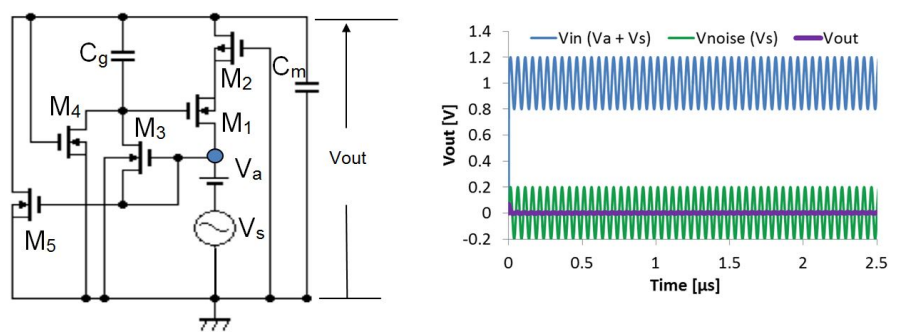

Fig. 13. Only Vs as noise source.
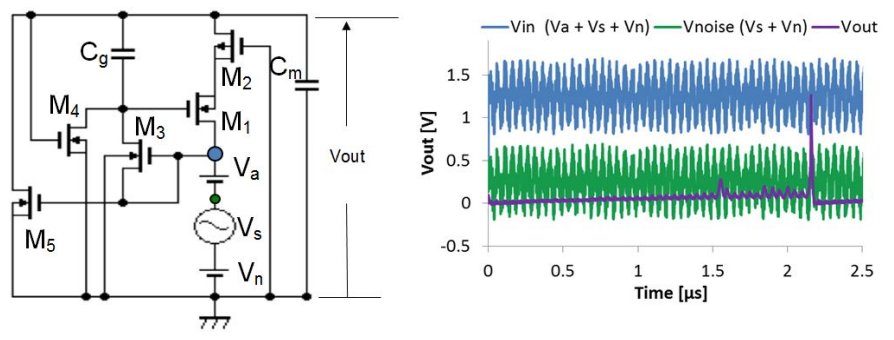

Fig. 14. Vs and Vn as noise sources.

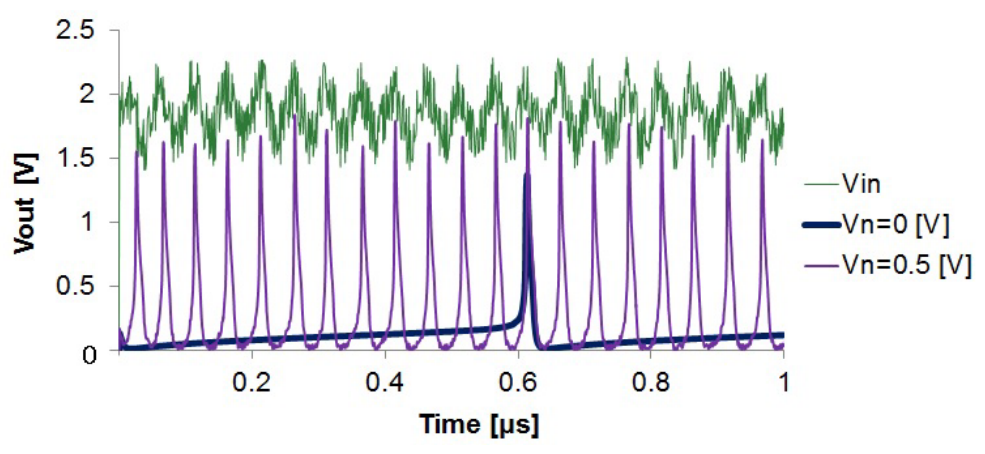

(a) Comparison of the output with and without noise

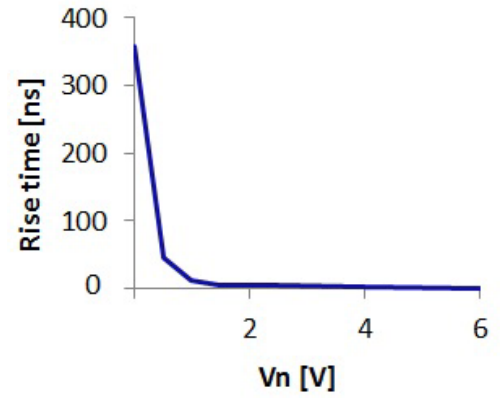

(b) Delay time characteristic for different values of noise

Fig. 15. Pulse-type cell body model output characteristic.

its characteristic pulse oscillation. This is due to the stochastic behavior of the circuit. For low noise values, the rise time is short and the amplitude is increased. For high noise values, the DC level in the output is elevated and the noise interference in the signal is visible. Therefore, the optimal amount of noise that should be applied is when the SNR has a peak; this SR characteristic is confirmed by the behavior of the circuit output. The presence of noise enables the model to oscillate, even at low 
values of Va. This can be observed in Fig. 16, where the horizontal axis represents the change in Va. The increase in the oscillation frequency due to the input is represented in the vertical axis. Here, $\mathrm{Vn}$ is $1[\mathrm{~V}]$. Figure 17 (a) depicts the output, when $\mathrm{Va}=\mathrm{Vn}=1[\mathrm{~V}]$. Here, noise enables the circuit to oscillate, even if the input (Va) is too low for oscillation. Figure 17(b) illustrates the output, when $\mathrm{Va}=1.6[\mathrm{~V}]$ and $\mathrm{Vn}=1[\mathrm{~V}]$. It demonstrates that the presence of noise enables an increase in the oscillation and a reduction in the time delay.

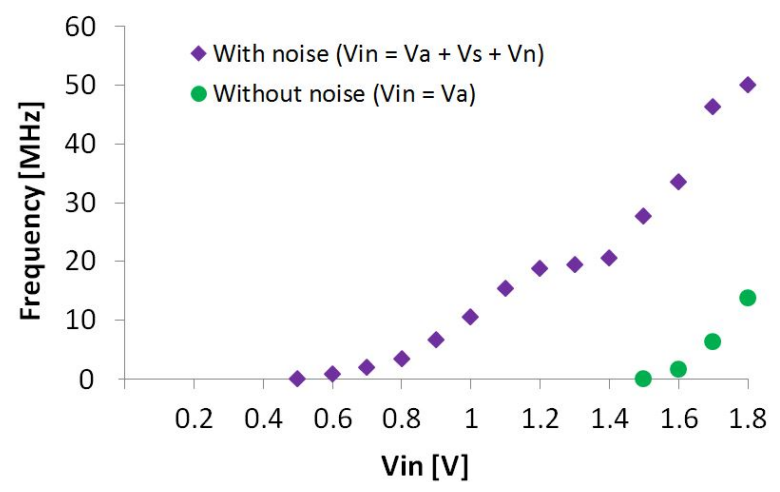

Fig. 16. Output for different values of Va.

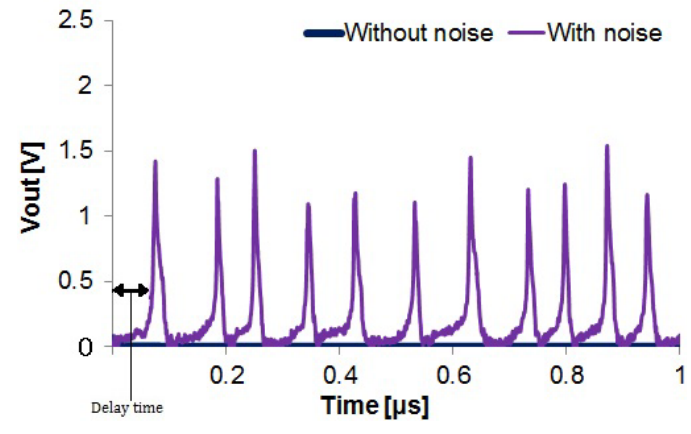

(a) $\mathrm{Vn}=1[\mathrm{~V}]$ and $\mathrm{Va}=1[\mathrm{~V}]$

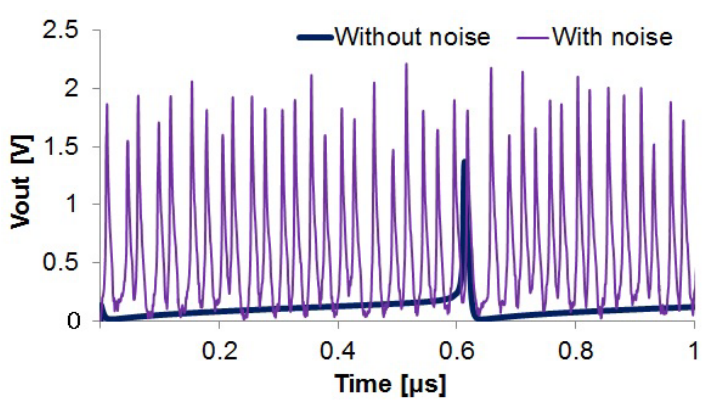

(b) $\mathrm{Vn}=1[\mathrm{~V}]$ and $\mathrm{Va}=1.6[\mathrm{~V}]$

Fig. 17. Pulse-type cell body model output.

\section{Discussion}

The noise used in this study has constant amplitude. It is also an evenly distributed random real number sequence. For another approach to the noise source, the evenly distributed random source, Vn, was replaced by a pink noise source, Vnpink. The signal was generated by the circuit shown in Fig. 18, where Vn represents the same PWL source added to the model previously.

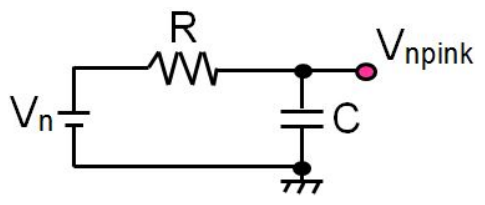

Fig. 18. Generation of pink noise (Vnpink).

The power spectrum obtained from the fast Fourier transform (FFT) of the circuit was calculated using the FFT measurement in HSPICE. The result is shown in Fig. 19, wherein, the power spectrum varies inversely, which is a characteristic of pink noise.

The SNR characteristic is shown in Fig. 20. It can be seen that for colored pink noise, the SNR does not demonstrate a characteristic SR bell shape. This may be because a high-level noise is required for the cell body model to oscillate. This condition is satisfied by noise with constant amplitude, such as white noise or in this case, evenly distributed random noise. 


\section{Vn (constant amplitude)}
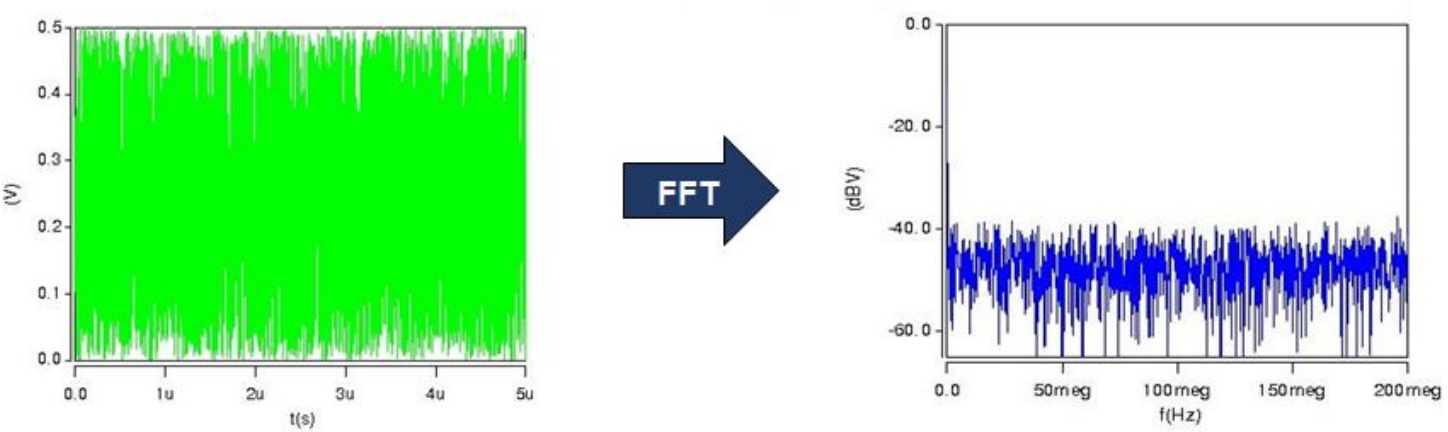

\section{$V_{\text {npink }}(1 / f$ amplitude)}

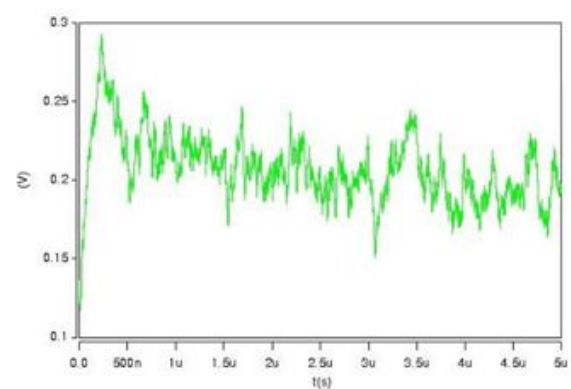

FFT

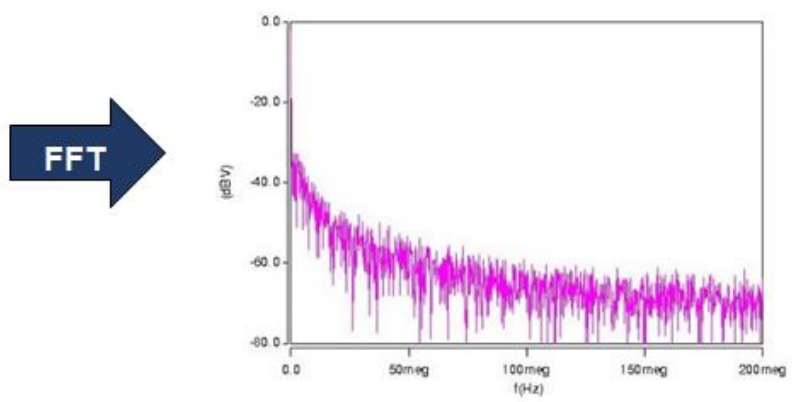

Fig. 19. Output of the pink noise source and FFT.

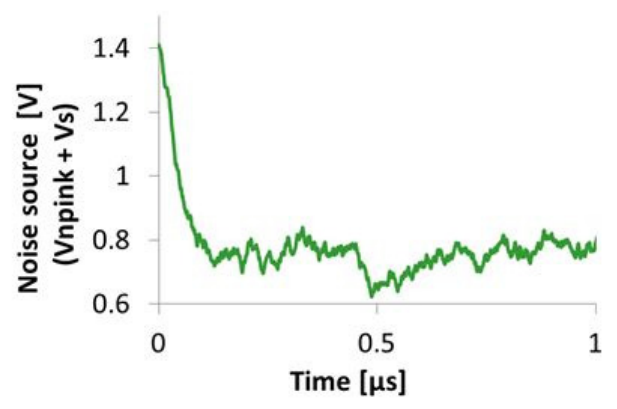

Fig. 20. Pink noise source output and its respective SNR characteristic.

\section{Conclusions}

The proposed model uses a $\Lambda$-shaped negative resistance that enables the characteristic pulse-type oscillation of the circuit. This oscillation is possible, when the circuit operates in the $\Lambda$-shaped negative resistance region. The study reveals that noise enables the circuit to active this region, benefiting the output oscillation. Therefore, to analyze the SR phenomenon, the changes in the output oscillation were analyzed. The results show that the addition of noise reduces the rise time of the output. This improvement is observed at any noise value and is linearly proportional to the amount of noise. However, when the noise exceeds the value where SNR has a peak, the output assumes the shape of the noise and loses its characteristic pulsed oscillation. Therefore, the SNR peak value indicates the correct amount of noise needed for enhancing the desired signal without damaging it. This outcome fulfills the principle of SR. Another benefit of noise in the model is that it reduces the input voltage needed to generate circuit oscillation.

It is observed that the SNR characteristic for pink noise does not demonstrate a characteristic SR bell shape. This may be because a high-level noise is required for the cell body model to oscillate. This condition is satisfied by noise with constant amplitude, such as white noise or in this case, evenly distributed random noise.

In future, for maximizing and better understanding the implication of adding noise to provide an application of the SR behavior in the pulse-type cell body model, it will be necessary to design a 
neuronal network that uses the benefits of noise such as fast data transmission and a high-threshold crossing output, found in this study. The characterization of this phenomenon will assist in understanding the processing of information by the human brain and lead the way for artificial neural systems.

\section{Acknowledgments}

The authors would like to acknowledge Research Assistant, Yoshiki Sasaki, from Nihon University for his invaluable discussions. This work was supported in part by the VLSI Design and Education Center (VDEC) of the University of Tokyo in collaboration with Cadence Design Systems, Inc.

\section{References}

[1] M.D. McDonnell and D. Abbott, "What is stochastic resonance? definitions, misconceptions, debates, and its relevance to biology," PLOS Computational Biology, vol. 5, no. 5, pp. 1-9, May 2009.

[2] P. Hänggi, "Stochastic resonance in biology how noise can enhance detection of weak signals and help improve biological information processing," ChemPhysChem, vol. 3, no. 3, pp. 285-290, March 2002.

[3] L.M. Ward, S.M. Doesburg, K. Kitajo, S.E. MacLean, and A.B. Roggeveen, "Neural synchrony in stochastic resonance, attention, and consciousness," Canadian Journal of Experimental Psychology/Revue canadienne de psychologie expérimentale, vol. 60, no. 4, pp. 319-326, December 2006.

[4] T. Mori and S. Kai, "Noise-induced entrainment and stochastic resonance in human brain waves," Physical Review Letters, vol. 88, no. 21, p.218101, May 2002.

[5] J.K. Douglass, L. Wilkens, E. Pantazelou, and F. Moss, "Noise enhancement of information transfer in crayfish mechanoreceptors by stochastic resonance," Nature, vol. 365, no. 6444, pp. 337-340, September 1993.

[6] D.F. Russell, L.A. Wilkens, and F. Moss, "Use of behavioural stochastic resonance by paddle fish for feeding," Nature, vol. 402, no. 6759, pp. 291-294, November 1999.

[7] A. Utagawa, T. Asai, and Y. Amemiya, "Stochastic resonance in simple analog circuits with a single operational amplifier having a double-well potential," NOLTA, vol. 2, no. 4, pp. 409-416, October 2011.

[8] H. Tanaka, T. Yamazato, Y. Tadokoro, and S. Arai, "Performance comparison of stochastic resonance receiver with Schmitt trigger, comparator, and three-level device for subthreshold signal reception," NOLTA, vol. 7, no. 3, pp. 407-418, July 2016.

[9] L. Gonzalez-Carabarin, T. Asai, and M. Motomura, "Application of nonlinear system for designing low-power logic gates based on stochastic resonance," NOLTA, vol. 5, no. 4, pp. 445-455, October 2014.

[10] M. Chatterjee and M.E. Robert, "Noise enhances modulation sensitivity in cochlear implant listeners: Stochastic resonance in a prosthetic sensory system?," Journal of the Association for Research in Otolaryngology, vol. 2, no. 2, pp. 159-171, June 2001.

[11] D. Querlioz and V. Trauchessec, "Stochastic resonance in an analog current-mode neuromorphic circuit," 2013 IEEE International Symposium on Circuits and Systems (ISCAS), pp. 1596-1599, May 2013.

[12] Y. Sekine and K. Saeki, "CMOS inplementation of a pulse-type hardware neuron model and its application," JNNS, vol. 15, no. 1, pp. 27-38, March 2008.

[13] Y. Sasaki, K. Saeki, and Y. Sekine, "Low capacity implementation of a pulse-type chaotic neuron model," IEEJ, vol. 136, no. 10, pp. 1424-1430, June 2016. 